EPJ Web of Conferences 59, 01009 (2013)

DOI: $10.1051 /$ epjconf/20135901009

(C) Owned by the authors, published by EDP Sciences, 2013

\title{
Advances in the national inertial fusion program of China
}

\author{
X.T. He ${ }^{a}$, W.Y. Zhang and the Chinese ICF team \\ Institute of Applied Physics and Computational Mathematics, Beijing 100088, China
}

\begin{abstract}
The planned inertial confinement fusion (ICF) ignition in China in around 2020 is to be accomplished in three steps. The first is carrying out target physics experiments in the existing laser facilities SG-II, SG-IIIP and SG-IIU (operating in 2012) of output energy 3-24 kJ at $3 \omega$. Results have been obtained for better understanding the implosion dynamics and radiation transport. Recent studies include efficiency of radiation generation, hydrodynamic instabilities, shock waves in cryogenic targets, opacity measurements using kJ lasers, etc. Hydrodynamic codes (the LARED series) have been developed and experimentally verified with over 5000 shots, and are applied to investigating target physics and ignition target design. For fast ignition, a large number of experiments and numerical simulations have led to improved understanding relevant to target design, hot electron transport, collimation by the spontaneous magnetic fields in overdense plasmas, etc. In addition to the SG-II, SG-IIU and SG-IIIP, the SG-III laser facility with energy of $200-400 \mathrm{~kJ}$ at $3 \omega$ shall operate in 2014 and be used for advanced target physics research. In the last step, the 1.5 MJ SG-IV laser facility still under design will be used to investigate ignition and burning.
\end{abstract}

\section{INTRODUCTION}

The national research institutes such as the Research Center of Laser Fusion (RCLF), the Institute of Applied Physics and Computational Mathematics (IAPCM), the National Laboratory on High Power Laser and Physics (NLHPLP), as well as many universities and other institutions are participating in the Inertial Confinement Fusion (ICF) Program of China. The program involves target physics (theory and experiment) and target fabrication, high-power lasers, and precision diagnostics. The roadmap towards ignition in around 2020 is being carried out in three steps. In the first step, target physics research and target design are based on the existing tens-kJ laser facilities in China. In the second step, pre-ignition target design and advanced target physics research, based on the SG-III laser facility, are crucial as the basis for the ignition target design in the coming six years, which should identify and solve the essential science and technology issues, to reduce risks, and to scale up the design to the ignition target. In the last step, ignition is to be demonstrated on the SG-IV laser ignition facility.

\section{DEVELOPMENT OF LASER DRIVERS}

The SG-II in operation has 8 beams with output energy of 3kJ (here and below, unless otherwise stated the laser energy is for $3 \omega$ ) and pulse duration of $1 \mathrm{~ns}$. Since 2000 it has provided over 3500 shots for experiments. The SG-IIU (SG-II upgrade) under construction for target physics research has an output energy of $24 \mathrm{~kJ}$ for the $3 \mathrm{~ns}$ pulse and should be operating in 2012. The petawatt (PW) laser with pulse duration of 1-5 picoseconds and output laser energy of $1 \mathrm{~kJ}$ at $1 \omega$ should also be operating in 2012. The SG-IIU combined with the PW laser will serve the fast ignition research.

\footnotetext{
ae-mail: xthe@iapcm.ac.cn
}

This is an Open Access article distributed under the terms of the Creative Commons Attribution License 2.0, which permits unrestricted use, distribution, and reproduction in any medium, provided the original work is properly cited. 
(a)

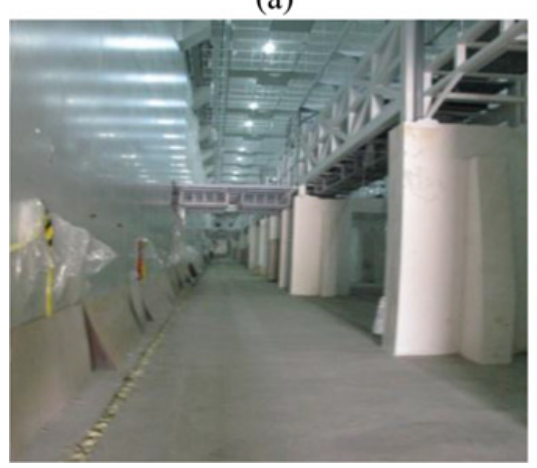

(b)

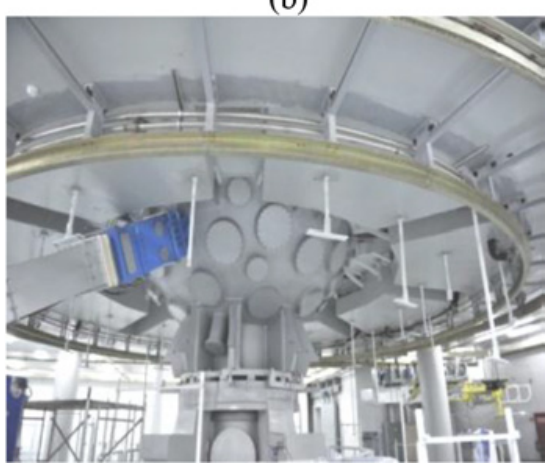

Figure 1. (a) SG-III laser bay. (b) The bottom of the target chamber, the operating-beam guide is in blue color.

An SG-III prototype (SG-IIIP) has energy output of $15 \mathrm{~kJ}$ with pulse duration of $3 \mathrm{~ns}$. Since 2006 it has completed over 1000 shots for experiments.

The SG-III facility (Figure 1a) under construction has 48 beams at output laser energy of 200-400 kJ with pulse duration of $3-5 \mathrm{~ns}$. The first bundle ( 8 beams) was installed in 2010 and the second bundle in 2010-2011. One beam (Figure 1b) with laser energy of $16 \mathrm{~kJ}$ at $1 \omega$ has been working since June 2011 for tests. Full laser energy output is expected in 2013 [1].

The SG-IV ignition facility is still being designed. The amount of laser energy required for ignition will be determined by scaling up the results from the target physics research on the SG-III. The SG-IV facility should be completed in around 2020.

\section{IMPROVED SIMULATION OF TARGET PHYSICS WITH LARED AND JASMIN}

JASMIN has an adaptive structure mesh application infrastructure. It builds a bridge among the LARED package, the parallel computers and the users. It allows for using non-structured multi-block mesh for complex geometries and Lagrangian mesh across material interfaces for dealing with large deformation, etc. It also improved indirect-drive simulations involving complicated geometries, multi-material and large deformations, parallel computing with several thousand processors.

The LARED series is a numerical code package for simulating direct- and indirect-drive ICF, and has been verified with about 5000 shots in experiments. A LARED-integrated code involving 2D hydrodynamics, radiation transport and multi-group diffusion, 3D ray tracing in the hohlraum, thermal conductivities for electrons and ions, and plasma burning, is also developed. Combined with JASMIN, LARED greatly improves simulations of indirect-drive ICF, and is being used in advanced research on target physics, especially of the ignition target.

\section{ADVANCES IN TARGET PHYSICS EXPERIMENTS (SELECTED EXAMPLES)}

M-band fraction. A novel flat-response x-ray detector for photon energies in the range $0.1-4 \mathrm{keV}$ has been applied to measure the radiation temperature $T$ and M-band emission from the hohlraum gold wall in the SG-II facility. For the M-band measurement, the system consists of the filter system $720 \mathrm{~nm} \mathrm{~B}$ and $3.9 \mu \mathrm{m} \mathrm{Sc}$ and $34 \mu \mathrm{m} \mathrm{Sc} \mathrm{(transmitting} \mathrm{area} \mathrm{18 \% ),} \mathrm{as} \mathrm{well} \mathrm{as} \mathrm{Al} \mathrm{cathode} \mathrm{XRD.} \mathrm{The} \mathrm{signal}$ $V=\int R(E) S(E, t) d E d \Omega=R S$, where $S(E, \Omega)$ is the spectrum of x-ray energy $E$ and solid angle $\Omega$, and $\mathrm{R}$ is a response function that approaches to a constant. Thus, we can obtain the measured x-ray flux $S=\int S(E, t) d E d \Omega$ when $R$ is calibrated. An M-band fraction of $\sim 6 \%$ was measured for laser energy of $2.1 \mathrm{~kJ}$ (MSE $~ 5 \%$ for $1.4-4.4 \mathrm{keV}$ ) at the SG-II facility [2]. 
(a)

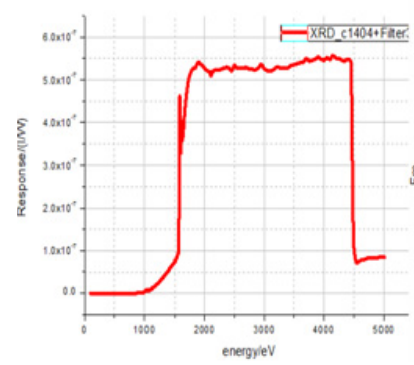

(b)

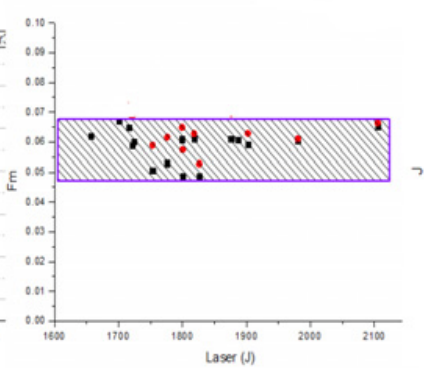

(c)

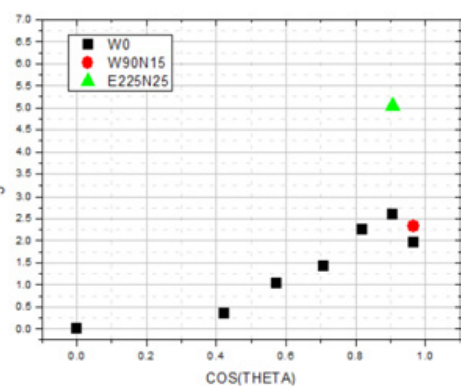

Figure 2. M-band emission from gold wall in the hohlraum as measured on the SG-II. (a) Response function $R$ vs x-ray energy. (b) M-band fraction vs laser energy. (c) Angular distribution of the M band.

(a)

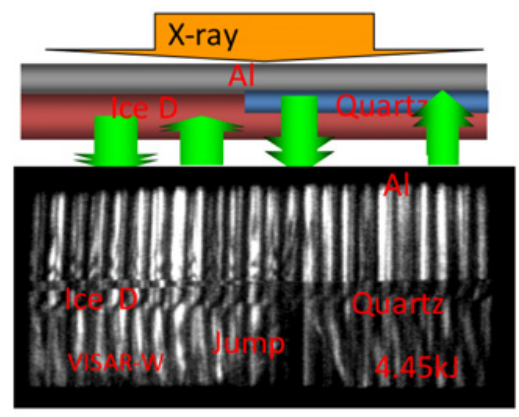

(b)

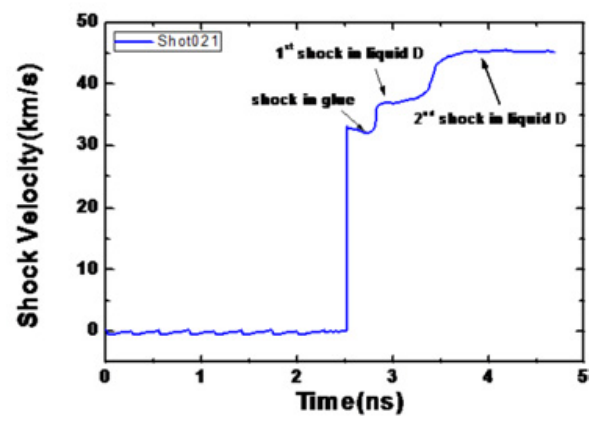

Figure 3. (a) The jumps of the first and second shocks in the iced deuterium can be clearly observed. (b) The velocity jumps are shown as the first one arrived and the second one catches up with the first.

Shock waves. Shocks are measured by VISAR (shock velocity resolution $\sim 2 \%$ ) on the SG-IIIP using cryogenic targets. A $4.45 \mathrm{~kJ}$ laser is incident on the inner wall of the hohlraum and the radiation ablates the $\mathrm{Al}$ foil and drives shock waves. The first shock propagates directly to the iced deuterium and the second one first penetrates the quartz and then into the iced deuterium. The second shock catches up with the first, as shown in Figure 3(a). The velocities of the two shocks vary with time, the shock passes through the glue interface between the $\mathrm{Al}$ and the quartz, causing a velocity change, as shown in Figure 3(b).

Opacity measurements. A new target configuration is used to measure opacity. The target is between two separated parts of the hohlraum and has a clean environment, where the scattering light and expanded plasmas from hohlraum are blocked by foam (Figure 4a). Thus, smaller hohlraum and lower laser energy can be used to achieve higher-temperature opacities without pollution. We have obtained Al opacity with $95 \mathrm{eV}$ radiation temperature at $1.5 \mathrm{~kJ}$ laser energy. The $\mathrm{Au}, \mathrm{Fe}$, and Ge opacities measured at $85 \mathrm{eV}$ radiation temperature (for $\rho \approx 20 \mathrm{mg} / \mathrm{cm}^{3}$ ) and $1.2 \mathrm{~kJ}$ laser energy are shown in Figures 4 (b)-(c). For measuring opacity of the dense target we have used x-ray scattering, as shown in Figures 4(d)-(f ) [3].

Measurements of the equation of state (EOS). Pressures of about $110 \mathrm{Mbar}$ for $\mathrm{Al}$ and about $230 \mathrm{Mbar}$ for $\mathrm{Au}$ are obtained from the measured shock velocity (SOP) by the step targets connected with the half-hohlraun. A laser of $6 \mathrm{~kJ}$ from SG-IIIP is incident on a small-size hohlraum of $\varnothing 700 \mu \mathrm{m} \times$ $585 \mu \mathrm{m}$. Simulations give the M-band fraction of $\sim 25 \%$ in such a hohlraum, where the energy from SRS has been deducted. The equivalent radiation temperature of $T=270-290 \mathrm{eV}$ is obtained from 
(a)

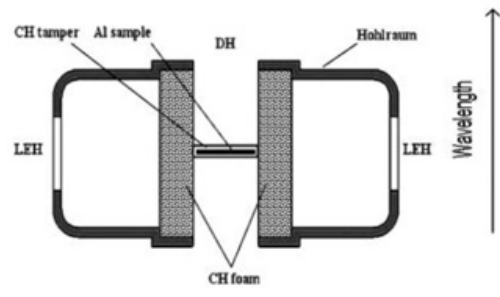

(d)

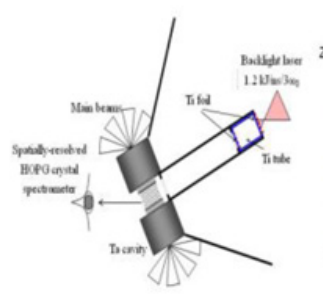

(b)

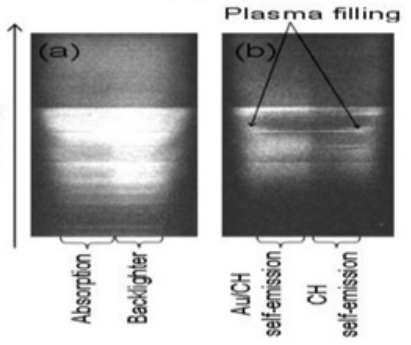

(e)
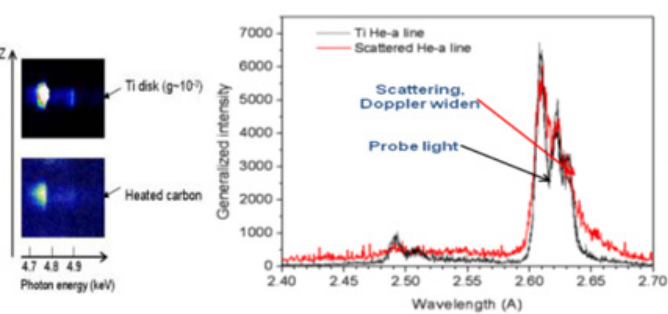

(c)

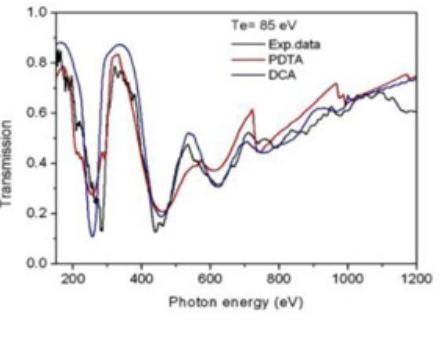

(f)

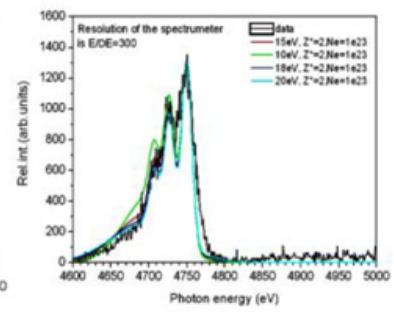

Figure 4. Measurements of opacity. (a) Clean hohlraum target, (b) absorption, backlight and self-emission spectrum measured using a time-gated grating spectrometer, (c) atomic physics calculation for opacity: DCA-detailed configuration model, PDTA-pseudo-detailed model, (d)-(e) bulk heating of carbon foam by Ta M-band x-ray, (f) comparison of experimental data and fits for various $T_{e}$.

(a)

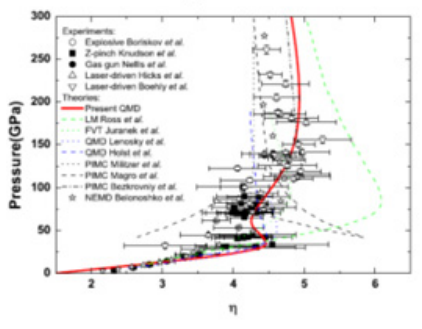

(b)

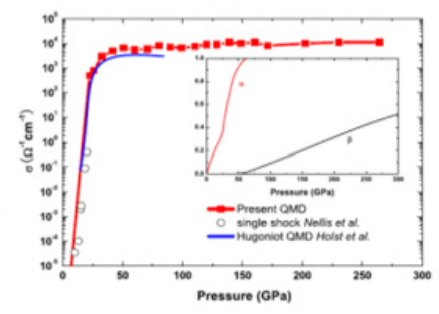

(c)

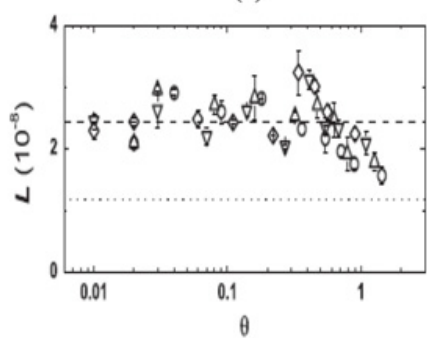

Figure 5. (a) Hugoniot curve for $\mathrm{D}$ for $<3 \mathrm{GPa}$, (b) electric conductivity $\sigma$ vs pressure, (c) Lorentz number vs $\theta$, for densities of $400(\mathrm{O}), 480(\Delta), 600(\nabla), 800(\diamond) \mathrm{g} / \mathrm{cm}^{3}$.

the scaling relation $T=\delta D \gamma$, where $\delta$ and $\gamma$ are adjustable parameters [4]. EOS for deuterium (D) for pressure less then 3 Mbar is simulated by QMD and OFMD coDEs. Results (red) by QMD [5] show maximal compressed ratio $\eta=4.5$ around $40 \mathrm{GPa}$ and $\eta=4.95$ about $180 \mathrm{GPa}$ due to D2 dissociation $(\alpha$, red) and D ionization ( $\beta$, black) as shown in Figure 5(a)-(b), and agree with the experiments. In the process of adiabatically compression of ICF, the fusion fuel and its reaction products are warm dense matter (WDM) under extremely high density and pressure, and are in partial degenerate states that have not a satisfactory theory to deal with. We have to apply massively parallel computing to investigate EOS. A Lorentz number of $L=\kappa / \sigma T$, where $\kappa$ and $\sigma$ are thermal and electric conductivities, respectively, is a constant of $2.44 \times 10^{-8}$ in the full degenerate state and $1.18 \times 10^{-8}$ in the non-degenerate state, which can be used to identify full degeneracy or not [6]. Numerical simulations show that for densities of 400$800 \mathrm{~g} / \mathrm{cm}^{3}$ the full degenerate theory is applicable for $\theta=T / T_{F} \leq 0.35$, and the partial degenerate theory for $0.35<\theta<2$, where $T_{F}$ is the Fermi temperature (see Figure 5(c)). 
(a)

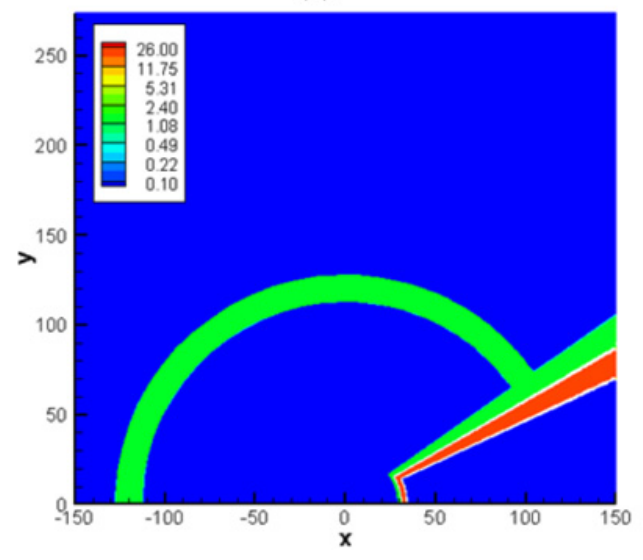

(b)

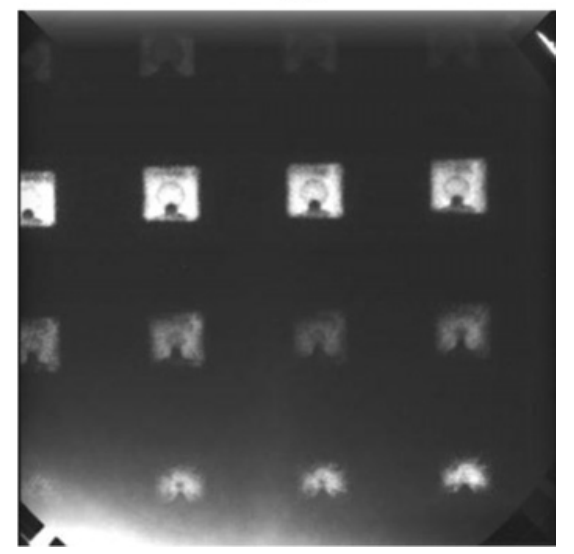

Figure 6. Simulation and experiment on fast ignition. (a) Surface of Au cone is coated by CD material, (b) indirectdrive implosion experiment on SG-II using Au cone-CD shell $(r \sim 250 \mu \mathrm{m})$ with hohlraum size $\varnothing 1000 \mu \mathrm{m} \times$ $1350 \mu \mathrm{m}$.

Fast ignition. Fast ignition is investigated by simulations and experiments. Implosion dynamics for the Au cone-CD shell targets shows that Au without coating is mixed into the CD plasma, but the mixing is suppressed with $\mathrm{CD}$ coating on the surface of the Au cone. It also shows that a guiding filament in double-cone tip generates spontaneous magnetic field that collimates the hot electron beam [7].

\section{REMARKS}

Since 2000, target physics research has been made through a series of experiments in the SG-II and SG-IIIP facilities, as well as extensive numerical simulations using the LARED-JASMIN codes. The ignition physics is also being advanced. Beginning 2012 the SG-IIU driver will serve experiments on the target physics, and will be coupled with the PW laser to investigate hotspot formation in fast ignition. The SG-III driver will be completed in about 2014. Target physics research on the SG-III is an important intermediate step towards ignition, and should find and solve relevant issues in the science and technology of eventual ignition. The 1.5 MJ SG-IV driver for direct ignition and high-gain fast ignition is in the design stage. China expects to attain moderate/high gain ignition and burning in about 2020.

\section{References}

[1] W. G. Zheng et al., Progress of the SG-III solid-state laser facility, presentation at IFSA2011, Bordeaux, France

[2] Z. C. Li et al., Rev. Sci. Instrum. 82, 106106 (2011)

[3] Jiyan Zhao et al., Phys. Rev. E 79, 016401 (2009); Yang Zhao, et al., Rev. Sci. Instrum. 80, 043505 (2009); Y. Xu et al., Phys. Plasmas 14, 052701 (2007)

[4] Yongsheng Li et al., Phys. Plasmas 18, 022701(2011)

[5] Cong Wang et al., J. Appl. Phys. 108, 044909 (2010)

[6] Cong Wang et al., Phys. Rev. Lett. 106, 145002 (2011)

[7] Hong-bo Cai et al., Phys. Rev. E 83, 036408 (2011); C. T. Zhou et al., Optics Lett. 36, 924 (2011) 